\title{
Update on Parkinson's Disease Therapy
}

\author{
Rebecca M Gilbert \\ American Parkinson Disease Association, Staten Island, NY, USA
}

DOI: https://doi.org/10.17925/USN.2021.17.2.92

S nce 2014, 11 new medications have been approved for the motor and non-motor symptoms of Parkinson's disease (PD), in addition to numerous advances in deep brain stimulation and the approval of focused ultrasound for certain PD indications. This article reviews these new options available for PD therapy and explains how best to utilize them in a treatment plan.

\section{Keywords}

Parkinson's disease, motor fluctuations, dyskinesias, non-motor symptoms

Disclosures: Rebecca M Gilbert is the Chief Scientific Officer at American Parkinson Disease Association and Clinical Associate Professor of Neurology at NYU Langone Health and Bellevue Hospital Center.

Review process: Double-blind peer review.

Compliance with ethics: This study involves a review of the literature and did not involve any studies with human or animal subjects performed by the author.

Data availability: Data sharing is not applicable to this article as no datasets were generated or analysed during the current study/during the writing of this article.

Authorship: The named authors meet the International Committee of Medical Journal Editors (ICMJE) criteria

for authorship of this manuscript, take responsibility for the integrity of the work as a whole, and have given final approval for the version to be published. Access: This article is freely accessible at touchNEUROLOGY.com. (C) Touch Medical Media 2021

Received: 10 October 2021

Accepted: 16 November 2021

Published online: 22 December 2021

Citation: touchREVIEWS in Neurology 2021;17(2):92-6

Corresponding author: Rebecca M Gilbert,

American Parkinson Disease Association, 135

Parkinson Avenue, Staten Island, NY 10305,

USA. E: rgilbert@apdaparkinson.org

Support: No funding was received in

the publication of this article.
Parkinson's disease (PD) is a progressive, neurodegenerative disorder that causes a range of motor and non-motor symptoms. The pathological hallmark of PD is the loss of dopaminergic neurons in the substantia nigra pars compacta of the midbrain and the presence of Lewy bodies, abnormal accumulations of the protein alpha-synuclein. Loss of dopaminergic neurons causes the motor symptoms that are typical of PD, including tremor, typically at rest, stiffness or rigidity of the muscles, slowness of movement or bradykinesia, and postural instability. A person with PD may also have a characteristic decrease in facial expression, a soft and monotonous voice, difficulty swallowing and trouble with posture and coordination. A stooped posture, with decreased heel strike, which gives the gait a shuffling quality, is characteristic. ${ }^{1}$

Of note, neuronal loss and Lewy bodies are features of many other brain regions in people with PD and are thought to be responsible for the non-motor symptoms of PD. Common non-motor symptoms of PD include cognitive dysfunction, hallucinations, orthostatic hypotension, sleep problems, constipation, anxiety, depression, fatigue and others. ${ }^{2}$

\section{Medications available prior to 2014}

Although first introduced in the 1960s, carbidopa/levodopa remains the most effective treatment for PD. ${ }^{3}$ Levodopa can cross the blood-brain barrier into the brain, where it is converted to dopamine. Levodopa reduces tremor, rigidity and bradykinesia in people with PD. The dopadecarboxylase inhibitor carbidopa, prevents levodopa from being broken down in the bloodstream before it reaches the brain. Carbidopa/levodopa has traditionally been available in immediaterelease and extended-release formulations. Side effects of carbidopa/levodopa include nausea, fatigue, orthostatic hypotension and hallucinations.

Other long-standing medications for PD include: ${ }^{4}$

- dopamine agonists, available in immediate-release, long-acting, patch form and injectable form (pramipexole, ropinirole, rotigotine, apomorphine)

- catechol-O-methyltransferase (COMT) inhibitors (entacapone, tolcapone)

- monoamine oxidase B (MAO-B) inhibitors (selegiline, rasagiline)

- anticholinergics (benztropine, trihexyphenidyl)

- amantadine.

Any discussion of PD therapies must highlight the important role that exercise, as well as physical, occupational and speech therapy, play in the management of PD motor and non-motor symptoms. ${ }^{5}$ Any treatment plan must incorporate exercise in order to maximize efficacy.

\section{Continued challenges of Parkinson's disease treatment}

Although the medications listed above brought substantial relief to motor symptoms of PD, additional therapies were necessary to address major impediments to quality of life as PD advances, including motor fluctuations, ${ }^{6}$ dyskinesias $^{7}$ and non-motor symptoms. ${ }^{8}$

\section{Motor fluctuations}

Motor fluctuations refer to disease and treatment-related difficulties that typically develop after several years of receiving PD therapy. OFF time is defined as segments of the day when medications do not maintain adequate symptom control. For some, OFF time can be unpredictable and/or sudden in onset, and not clearly related to timing of doses. 
Adjusting the amount and timing of baseline medications and adding supplemental medications were available strategies to reduce OFF time. However, OFF time remained a difficult problem for many, prompting the development of additional medications to improve this problem.

\section{Dyskinesias}

Levodopa treatment can lead to the development of dyskinesias, especially at the time of peak brain dopamine levels or when brain dopamine levels are in flux. Dyskinesias may not be troublesome, and many people with PD report they prefer some dyskinesia with good motor symptom control to no dyskinesia with less complete symptom control. Sometimes, however, dyskinesias can be socially distressing and/or disabling. New treatments were therefore necessary to address dyskinesias.

\section{Non-motor symptoms}

The established medications for PD almost exclusively sought to treat the motor symptoms of PD, despite the fact that as PD progresses, nonmotor symptoms typically become more and more problematic, eclipsing the motor symptoms in their effect on quality of life. Typically, as motor symptoms worsen, non-motor symptoms worsen too. In addition, dopaminergic medications can worsen some non-motor symptoms such as hallucinations and orthostatic hypotension. ${ }^{9}$ Some studies, however, suggest that dopaminergic medications may improve non-motor symptoms. ${ }^{10}$

New treatments sought to treat non-motor symptoms that had not previously been the focus of PD-specific therapy. The remainder of this article highlights new medications approved in the USA since 2014. This author recognizes that some of these medications are not available in many countries, and other medications are available in some countries, but not in the USA. However, a comprehensive discussion of worldwide PD medications is beyond the scope of this article.

\section{Medications for treatment of motor fluctuations}

A number of new medications approved since 2014 have been designed to treat motor fluctuations and reduce OFF time. These medications fall into four major categories:

- new formulations of carbidopa/levodopa that last longer than an immediate-release carbidopa/levodopa dose

- other medications that lengthen the effect of a carbidopa/levodopa dose

- new formulations of carbidopa/levodopa that are used as needed if medication effects wear off

- other medications that are used as needed if medication effects wear off.

In general, new medications that extend the length of a carbidopa/ levodopa dose are used if OFF time is somewhat predictable and occurs prior to next dose. New medications that are used as needed are most beneficial when OFF time is not predictable.

\section{New formulations of levodopa that last longer than immediate release}

\section{Carbidopa/levodopa extended-release capsules (Rytary ${ }^{\star}$ )}

Carbidopa/levodopa extended-release capsules (Rytary ${ }^{\circledR}$; Amneal Pharmaceuticals, Inc., Bridgewater, NJ, USA) was approved in 2015, and is a formulation of carbidopa/levodopa that is designed to prolong the effects of carbidopa/levodopa. The capsules contain both immediaterelease and extended-release beads, which are of different sizes and dissolve at different rates. Capsules can be opened and added to food without losing their extended-release properties.
Multiple clinical trials were conducted with carbidopa/levodopa before approval. ${ }^{11}$ The APEX-PD trial compared three doses of carbidopa/ levodopa extended-release capsules and demonstrated its efficacy over placebo in early PD. ${ }^{12}$ Advance-PD was a phase III randomized, double-blind trial, which demonstrated improvement in OFF time by an average of 1.17 hours with extended-release capsules compared with immediate release. ${ }^{13}$

Transitioning from immediate-release carbidopa/levodopa to extendedrelease capsules can be challenging but rewarding. In this author's clinical experience, if insufficient extended-release capsules are prescribed, patients may stop the medication because they find it is not helpful. This observation is supported by a published expert discussion suggesting that patients be switched to a total daily dose of extended-release carbidopa/levodopa capsules equivalent to $1.5-2$ times the total daily dose of immediate-release carbidopa/levodopa divided into three to four doses. ${ }^{14}$ Modifications of this dose may be necessary over subsequent weeks. Four strengths of the carbidopa/levodopa extended-release capsules are available: $23.75 / 95,36.25 / 145,48.75 / 195,61.25 / 245$, which allows the dose to be refined.

\section{Carbidopa/levodopa enteral suspension (Duopa ${ }^{\mathrm{TM}}$ )}

Carbidopa/levodopa enteral suspension (Duopa'TM; AbbVie Inc., North Chicago, IL, USA) was approved by the US Food and Drug Administration (FDA) in 2015. It is infused directly into the gut through a percutaneous endoscopic transgastric jejunostomy (PEG-J) tube. This allows levodopa to be delivered continuously into the jejunum, smoothing out its blood and brain levels. A double-blind, randomized clinical trial demonstrated that mean OFF time decreased by 2 hours, and mean ON time without troublesome dyskinesias increased by almost 2 hours in patients receiving the enteral suspension compared with those receiving oral doses. ${ }^{15}$ Two other randomized controlled trials support these findings. ${ }^{16,17}$

The levodopa suspension is stored in a cartridge that is attached to a wearable pump. The pump can fit in a vest or waist bag and can be programmed to deliver a wide range of infusion rates as well as boluses of medication as needed. The medication is delivered up to 16 hours a day. Complications, including infection of the PEG-J insertion site, are possible. Additionally, the tube may clog, kink or become dislodged.

The side effects of the carbidopa/levodopa infusion are similar to those of oral carbidopa/levodopa, but may be associated with a higher incidence of peripheral neuropathy. ${ }^{18}$

\section{New medications that lengthen the effect of a dose of carbidopa/levodopa Istradefylline (Nourianz)}

Istradefylline (Nourianz ${ }^{\circledR}$; Kyowa Kirin Inc., Bedminster, NJ, USA) is an adenosine A2A receptor antagonist, which was approved in the USA in 2019 as an add-on therapy to levodopa for treatment of OFF time in PD. It has a novel mechanism of action, acting on the adenosine receptor, which modulates the dopaminergic system, but is not directly dopaminergic. It is the first medication in its class to be approved for PD. The drug was developed in Japan and underwent clinical trials in both Japan and the USA. The initial clinical trials conducted in the USA, ${ }^{19-21}$ collectively showed a reduction in OFF time of about 0.7 hours per day. These trials were evaluated by the FDA in 2008, which did not approve its use due to concern that the motor improvement shown in the trials was too small and not clinically significant. Further trials were then conducted, ${ }^{22}$ and based on the newer data, the FDA did approve its use in 2019. 


\section{Opicapone (Ongentys ${ }^{\circledR}$ )}

Opicapone (Ongentys ${ }^{\oplus}$; Bial Pharma UK Ltd., Windsor, UK; licensed in the USA by Neurocrine Biosciences Inc, San Diego, CA, USA) is a COMT inhibitor that is taken once a day. It was approved in the USA in 2020 as an add-on therapy to levodopa for motor fluctuations. Its approval was based on two randomized, placebo-controlled phase III trials, BI-PARK $1^{23}$ and BI-PARK $2{ }^{24}$ Both studies demonstrated similar results. Opicapone $25 \mathrm{mg}$ and $50 \mathrm{mg}$ significantly reduced OFF time compared with placebo. BI-PARK 1 included an arm of patients taking entacapone 200 mg, an FDA-approved COMT inhibitor that is taken with each dose of levodopa, and demonstrated that opicapone was not worse than entacapone, and in fact showed a greater magnitude of reduction in OFF time. While the placebo group demonstrated a change in OFF time of 56 minutes, and entacapone demonstrated a change in OFF time of 96 minutes, opicapone $50 \mathrm{mg}$ demonstrated a change in OFF time of 117 minutes. Long-term efficacy data were recently published ${ }^{25}$ and demonstrated maintenance of good effect without an increase in troublesome dyskinesias.

\section{Safinamide (Xadago ${ }^{\circledR}$ )}

Safinamide (Xadago ${ }^{\oplus}$; Newron Pharmaceuticals, Milan, Italy, manufactured in the USA by Supernus, Rockville, MD) was approved in 2017 as an add-on therapy to carbidopa/levodopa for the treatment of OFF time. ${ }^{26}$ It is a selective MAO-B inhibitor but has other mechanisms of action such as inhibition of glutamate release. Clinical trials demonstrated increased ON time in PD patients with motor fluctuations, with the placebo group demonstrating an improvement of 0.57 hours and safinamide demonstrating an improvement of 1.42 hours. $^{27-29}$

\section{New formulations of levodopa designed to be used as needed Levodopa inhalation powder (Inbrija®)}

Levodopa inhalation powder (Inbrija ${ }^{\circledast}$; Acorda Therapeutics, Inc., Ardsley, NY, USA) was approved in 2018 to be used as needed if medication effects wear off between oral doses of carbidopa/levodopa. ${ }^{30}$ A phase III trial showed that the treatment improved United Parkinson's Disease Rating Scale (UPDRS) motor scores during OFF periods, with few serious adverse effects. The change in UPDRS from pre-dose to 30 minutes post-dose was 5.91 for the placebo group and 9.83 for the inhaled Levodopa group. ${ }^{31}$

A breath-activated inhalation device, is provided with the medication into which capsules of $42 \mathrm{mg}$ of levodopa are inserted. One dose consists of two capsules. Absorption of the medication is through the pulmonary tree, which bypasses the gastrointestinal tract. The capsules only contain levodopa without a dopa-decarboxylase inhibitor, which means that the inhalation powder needs to be added to a regimen of carbidopa/levodopa.

In this author's clinical experience, a potential limitation of the system is that the device cannot be pre-loaded. Therefore, the capsules need to be unwrapped and inserted into the inhalation device when the patient if OFF and has decreased dexterity.

\section{Other medications used as needed if medication effects wear off} Apomorphine $\mathrm{HCl}$ sublingual film (Kynmobi®)

Apomorphine is a dopamine agonist with rapid onset of effect. It can be used as a rescue medication for someone whose dose of levodopa wears off unexpectedly before the next dose is due. Previously, apomorphine was dispensed as an under-the-skin injection. In 2020, a sublingual film was approved (Kynmobiø; Sunovion, Marlborough, MA, USA, a whollyowned subsidiary of Sumitomo Dainippon Pharma Co.), which can be used as needed during the day if medication effects wear off. Clinical trials demonstrated that sublingual apomorphine was an effective treatment for OFF episodes. ${ }^{32,33}$ The change in UPDRS from pre-dose to 30 minutes post-dose was 3.5 for the placebo group and 11.1 for the sublingual apomorphine group. ${ }^{34}$

This new route of delivery is associated with oropharyngeal side effects, including dry mouth, oral mucosal irritation and swelling, and throat irritation, which can lead to discontinuation of treatment. ${ }^{35}$ In addition, apomorphine (both injectable and sublingual) can induce nausea, leading to the recommendation that it be taken with an antiemetic, typically trimethobenzamide.

\section{Medications for treatment of dyskinesia Amantadine}

Originally used to prevent or treat influenza, amantadine was observed to ease the tremor of PD as well as muscle rigidity. It has therefore been used as an adjunct medication to other therapies for PD. In addition, it was also observed to be effective at decreasing dyskinesias caused by levodopa and is typically prescribed two to three times a day. ${ }^{36}$

In 2017, an extended-release formulation of amantadine (Gocovri ${ }^{\text {* }}$; formerly owned by Adamas Pharmaceuticals, Inc., Emeryville, CA, USA, now Supernus, Rockville, MD, USA), was approved. It is taken once daily at night, and is indicated for both the treatment of levodopa-induced dyskinesias and for reduction of OFF time. It is designed to provide an initial lag, followed by a slow rise in amantadine concentration during the night, and a high concentration in the morning and through the waking day. Results of two phase III clinical trials demonstrated improvement in both dyskinesias and OFF time. ${ }^{37,38}$ In 2018, a second extended-release formulation of amantadine (Osmolex ER ${ }^{\mathrm{TM}}$; formerly owned by Osmotica Pharmaceuticals, Bridgewater, NJ, USA, now Supernus, Rockville, $M D$, USA ${ }^{39}$ was approved. It is taken once daily in the morning and is indicated for the treatment of PD motor symptoms as well as druginduced parkinsonism.

\section{Medications for treatment of non-motor symptoms} Treatment of neurogenic orthostatic hypotension

Neurogenic orthostatic hypotension ( $\mathrm{NOH}$ ) is a common non-motor symptom in PD and can be a major driver of disability, causing chronic dizziness and contributing to falls. Other symptoms that can be correlated to $\mathrm{NOH}$ include weakness, fatigue and cognitive slowing. ${ }^{40}$ $\mathrm{NOH}$ can be a symptom of PD itself as well as a side effect of many PD medications, including levodopa. Although first steps in management involve implementing lifestyle changes (such as increasing dietary fluids and salts) and removing exacerbating medications, prescription medication may be necessary to maintain blood pressure. Midodrine is approved for orthostatic hypotension and is often used in clinical practice. Fludrocortisone is also often used, although not specifically approved for this indication. ${ }^{41}$

\section{Droxidopa (Northera'M ${ }^{\text {TM }}$}

Droxidopa (Northera'm; formerly owned by Chelsea Therapeutics, Charlotte, NC, USA, now Lundbeck, Deerfield IL, USA) was approved in 2014 for the treatment of $\mathrm{NOH}$ caused by the autonomic failure of PD and other diseases.

Droxidopa is a synthetic amino acid analogue that is metabolized to norepinephrine by amino acid decarboxylase. Once metabolized into norepinephrine, it increases blood pressure by inducing peripheral 
arterial and venous vasoconstriction. Results of four phase III trials were contradictory, with two meeting their primary outcomes of improved symptom control and raised blood pressure.42-45 Studies also utilized the Orthostatic Hypotension Questionnaire, a validated symptom assessment scale. ${ }^{45}$ Despite mixed results, the studies led to FDA approval. ${ }^{47}$ Approval was granted under the Accelerated Approval Program, provided that further studies would be conducted to verify the clinical effects.

Supine hypertension is a concern for all medications given for $\mathrm{NOH}$ and doses should not be given close to bedtime.

\section{Treatment of psychosis}

Psychosis (encompassing hallucinations and delusions) is another common non-motor symptom of PD and can significantly affect quality of life. The first step in management is to review all medications and lower or stop any potential offenders. Most PD medications can contribute to hallucinations, so lowering these medications must be considered, but must also be balanced with its impact on motor symptoms. ${ }^{48}$

Most antipsychotic medications act by blocking postsynaptic dopamine receptors and can therefore causes parkinsonism. Two antipsychotics, quetiapine and clozapine, can be used safely, but off-label, for Parkinson's disease psychosis (PDP). Clinical trial data for quetiapine are lacking, but there are clinical trial data for the use of clozapine. ${ }^{49}$ However, because clozapine carries a known risk of agranulocytosis and requires regular blood monitoring, it is difficult to use in practice. ${ }^{49}$

\section{Pimavanserin (Nuplazid ${ }^{\circledR}$ )}

Pimavanserin (Nuplazid ${ }^{\oplus}$; Acadia Pharmaceuticals Inc., San Diego, CA, USA) is a selective serotonin inverse agonist, which was approved in 2016 as a treatment for PDP. A randomized placebo-controlled phase III trial demonstrated modest clinical improvement on a novel PDP scale called the Scale for the Assessment of Positive Symptoms for Parkinson's Disease Psychosis..$^{50}$ Because the scale had not been widely used previously, the decision of the FDA engendered some controversy. ${ }^{51}$ Pimavanserin remains the only medication specifically approved for PDP. Recently published real-world clinical data demonstrated clinical improvement in $76 \%$ of patients, with adverse effects reported in $22 \% .^{52}$

Of note, pimavanserin, as well as other antipsychotic medications, carry a black-box warning of increased risk of death in elderly patients with dementia-related psychosis associated with the use of these drugs. After approval, concern was raised that the risk of death with pimavanserin exceeded that of other antipsychotics, but a review by the FDA concluded in 2018 that post-marketing data were consistent with safety data obtained during clinical trials.53

\section{Procedures for Parkinson's disease: Deep brain stimulation and high-intensity focused ultrasound}

Despite the multiple medication options available, motor fluctuations and dyskinesias can remain difficult problems. In addition, certain patients with PD do not gain adequate control of their tremor with medications. When medication management does not achieve sufficient symptom control, a neurosurgical procedure such as deep brain stimulation (DBS) or high-intensity focused ultrasound (HIFU) may be indicated.

\section{Deep brain stimulation}

DBS is a neurosurgical procedure for people with PD who retain a good response to levodopa, but who have developed significant motor fluctuations including dyskinesias. DBS may also be used to treat medication-resistant tremor. DBS involves the implantation of permanent, thin electrodes into selected deep parts of the brain. Contacts along the electrodes deliver electricity to very targeted areas of the brain. A battery-operated pulse generator is implanted under the skin of the chest or abdomen.

DBS technology is constantly evolving with many new developments in recent years. ${ }^{54}$ Certain systems offer rechargeable batteries. The newer DBS systems are magnetic resonance imaging (MRI) conditional and can be placed in MRI mode or turned off for the duration of an MRI.

In the past, the surgery was only performed when a patient was awake (albeit under mild sedation and with appropriate pain control), allowing the surgeon to monitor symptoms as the electrode was advanced. Certain surgeons will now perform the surgery in the bore of a computed tomography scanner or MRI machine, allowing for visualization of the electrode. When performed this way, the patient can be completely asleep during the procedure.

Although DBS is typically performed at more advanced stages of disease, the FDA has also approved DBS for earlier-stage PD of at least 4 years' duration with motor symptoms that are not adequately controlled with medication, including recent-onset motor complications.

The available DBS systems for PD are all slightly different. Each offers its own technology in programming the stimulation variables, with the goal of maximizing symptom control and minimizing the side effects of stimulation. All the systems can utilize directional leads, in which the contacts on the electrodes are subdivided into sections around the circumference of the electrode and stimulation can be applied to different sections. This allows for the field of stimulation to be steered in a particular direction, with the goal of reducing side effects of stimulation. ${ }^{55}$ Clinical trials have demonstrated the clinical benefit of this approach..$^{56,57}$

\section{Abbott}

The Infinity ${ }^{\mathrm{TM}}$ system (Abbott Laboratories, Abbott Park, IL, USA) uses directional leads. Abbott also offers Neurosphere ${ }^{\mathrm{TM}}$, a remote programming option that allows clinicians to connect with patients through a wireless network and perform DBS programming through a telemedicine appointment.

\section{Boston Scientific}

The Vercise ${ }^{\mathrm{TM}}$ system (Boston Scientific, Marlborough, MA, USA) offers two different electrodes, one with eight contacts, more than other leads, and one that offers directional stimulation. The electrodes use multiple independent current control, which means that each contact has its own energy source and allows for a more customizable field of stimulation.

\section{Medtronic}

The SenSight'M system (Medtronic, Minneapolis, MN, USA) uses directional leads, allowing for a customized field of stimulation. The SenSight ${ }^{\mathrm{TM}}$ system is compatible with the Percept ${ }^{\mathrm{TM}}$ PC Neurostimulator with Brainsense ${ }^{\mathrm{TM}}$ system (Medtronic), which captures local field potentials (LFPS) from the neurons that are in contact with the DBS electrode. DBS adjustments can be made based on LFP patterns of an individual patient as they correlate with reported symptoms and medication ingestion. Clinical studies are under way to determine whether adjustment based on LFP patterns improves outcomes. 


\section{High-intensity focused ultrasound}

HIFU is a procedure that uses targeted ultrasound waves to create lesions in a deep brain structure. HIFU of the ventral intermediate nucleus of the thalamus was approved in 2018 for the treatment of tremor in PD based on a randomized clinical trial, ${ }^{58}$ which demonstrated a $62 \%$ improvement of on-medication tremor score after HIFU of the ventral intermediate nucleus compared with a $22 \%$ improvement in the group that received the sham procedure. Side effects included mild and reversible hemiparesis, as well as parasthesias and one case of ataxia. Exablate Neuro ${ }^{\circledR}$ is the focused ultrasound platform designed for brain treatments (Insightec, Miami, FL, USA).

HIFU of the globus pallidus interna has very recently (November 2021) been approved for the treatment of bradykinesia, rigidity and dyskinesias associated with PD. Data from the pivotal trial that led to its approval have not yet been published, but published data from an open-label clinical trial demonstrated improvements in dyskinesia scores and UPDRS scores from baseline. ${ }^{59}$

\section{Conclusions}

With multiple new medications and procedures available for the treatment of $\mathrm{PD}$, there is more hope than ever that symptoms can be successfully managed for many years of PD. However, even with the new treatments discussed above, PD presents significant challenges for many people, especially as the disease advances. Research focused on improvement of motor and non-motor symptoms continues. In addition, discovering treatments that will slow down or halt disease progression is a major emphasis of current PD research, with the hope that soon medications will be available not only to treat symptoms, but also to improve the course of PD. $\square$
Jankovic J. Parkinson's disease: clinical features and diagnosis. Neurol Neurosurg Psychiatry. 2008;79:368-76.

2. Balestrino R, Schapira AHV. Parkinson disease. Eur J Neurol. $2020 \cdot 27 \cdot 27-42$.

3. Armstrong MI. Okun MS. Diagnosis and treatment of Parkinson disease: a review. JAMA. 2020;323:548-60.

4. Tarakad A, Jankovic J. Diagnosis and management of Parkinson's disease. Sem Neurol. 2017;37:118-26.

5. Ellis T, Rochester L. Mobilizing Parkinson's disease: the future of exercise. J Parkinson Dis. 2018;8(s1):s95-s100.

6. Aradi SD, Hauser RA. Medical management and prevention of motor complications in Parkinson's disease. Neurotherapeutics. 2020;17:1339-65.

7. Pilleri M, Antonini A. Therapeutic strategies to prevent and manage dyskinesias in Parkinson's disease. Expert Opin Drug Saf. $2015 ; 14: 281-94$

8. Schapira AHV, Chaudhuri KR, Jenner P. Non-motor features of Parkinson disease. Nat Rev Neurosci. 2017:18:435-50.

9. Schaeffer E, Berg D. Dopaminergic therapies for non-motor symptoms in Parkinson's disease. CNS Drugs. 2017:31:551-70.

1. Muller T. TANIMOS Study Investigators. Tolcapone addition improves Parkinson's disease associated nonmotor symptoms. improves Parkinson's disease associated

11. Muller T, Mohr JD. Efficacy of carbidopa-levodopa extendedrelease capsules (IPX066) in the treatment of Parkinson disease. Expert Opin Pharmacother. 2018;19:2063-71.

12. Pahwa R, Lyons KE, Hauser RA, et al. Randomized trial of IPX066, carbidopa/levodopa extended release, in early Parkinson's disease. Parkinsonism Relat Disord. 2014;20:142-8.

13. Hauser RA, Hsu A, Kell S, et al. Extended-release carbidopalevodopa (IPX066) compared with immediate-release carbidopa-levodopa in patients with Parkinson's disease and motor fluctuations: a phase 3 randomised, double-blind tria Lancet Neurol. 2013:12:346-56.

14. Silver D, Trosch RM. Physicians' experience with RYTARY (carbidopa and levodopa) extended-release capsules in patients who have Parkinson disease. Neurology. 2016;86(14 Suppl 1):S25-S35.

15. Olanow CW, Kieburtz K, Odin P, et al. Continuous intrajejunal infusion of levodopa-carbidopa intestinal gel for patients with advanced Parkinson's disease: a randomised, controlled, double-blind, double-dummy study. Lancet Neurol. 2014;13:141-9.

16. Nyholm, D, Askmark H, Gomes-Trolin C, Knutson T, Lennernas $H$, Nystrom C, Aquilonius SM. Optimizing levodopa pharmacokinetics: intestinal infusion versus oral sustainedrelease tablets. 2003. Clin Neuropharmacol. 26: 156-63

17. Nyholm D, Nilsson Remahl Al, Dizdar N, Constantinescu R, Holmberg $B$ Jansson $R$, et al. Duodenal Covodopa infusion monsion monotherapy vs oral polypharmacy in

18. Merola A, Romagnolo A, Zibetti M, et al. Peripheral neuropathy associated with levodopa-carbidopa intestinal infusion: a longterm prospective assessment Eur J Neurol. 2016:23:501-9.

19. Hauer RA, Shulman LM, Trugman JM, et al. Study of istradefylline in patients with Parkinson's disease on levodopa with motor fluctuations. Mov Disord. 2008;23:2177-85.

20. Stacy M, Silver D, Mendis T, et al. A 12-week, placebo-controlled study (6002-US-006) of istradefylline in Parkinson disease. Neurology. 2008;70:2233-40.

21. LeWitt PA, Guttman $M$, Tetrud JW, et al. Adenosine A2A recepto antagonist istradefylline (KW-6002) reduces "off" time in Parkinson's disease: a double-blind, randomized, multicenter clinical trial (6002-US-005). Ann Neurol. 2008;63:295-302.

22. Berger AA, Winnick A, Welschmeyer $A$, et al. Istradefylline to treat patients with Parkinson's disease experiencing "Off" episodes: a comprehensive review. Neurol Int. 2020;12:109-29.

23. Ferreira JJ, Lees AJ, Rocha JF, et al. Opicapone as an adjunct to levodopa in patients with Parkinson's disease and end-of-dose motor fluctuations: a randomised, double-blind, controlled tria Lancet Neurol. 2016;15:154-65.

24. Lees A Ferreira 1 . Rascol $O$ et al. Opicapone as adjunct to levodopa therapy in patients with Parkinson disease and motor fluctuations: a randomized clinical trial. JAMA Neuro 2017;74:197-206

25. Ferreira JJ, Lees AJ, Rocha JF, et al. Long-term efficacy of opicapone in fluctuating Parkinson's disease patients: a poole analysis of data from two phase 3 clinical trials and their openlabel extensions. Eur J Neurol. 2019;26:953-60.

26. Blair HA, Dhillon S. Safinamide: a review in Parkinson's disease CNS Drugs. 2017:31:169-76.

27. Schapira AHV, Fox SH, Hauser RA. Assessment of safety and efficacy of safinamide as a levodopa adjunct in patients with Parkinson disease and motor fluctuations: a randomized Clinical trial. JAMA Neurol. 2017:74:216-24.

28. Borgohain R Szasz t Stanzione P et al Randomized trial of safinamide add-on to levodopa in Parkinson's disease with motor fluctuations. Mov Disord. 2014;29:229-37.

29. Borgohain R, Szasz J, Stanzione P, et al. Two-year, randomized, controlled study of safinamide as add-on to levodopa in mid to late Parkinson's disease. Mov Disord. 2014;29:1273-80.

30. Paik J. Levodopa inhalation powder: a review in Parkinson's disease. Drugs. 2020;80:821-8.

31. LeWitt PA, Hauser RA, Pahwa R, et al. Safety and efficacy of CVT-301 (levodopa inhalation powder) on motor function during off periods in patients with Parkinson's disease: a randomised, double-blind, placebo-controlled phase 3 trial. Lancet Neurol. 2019;18:145-54.

32. Katzenschlager R, Poewe W, Rascol O, et al. Apomorphine subcutaneous infusion in patients with Parkinson's disease with persistent motor fluctuations (TOLEDO): a multicentre, double-blind, randomised, placebo-controlled trial. Lancet Neurol. 2018:17:749-59.

33. Olanow CW, Factor SA, Espay AJ, et al. Apomorphine sublingua film for off episodes in Parkinson's disease: a randomised, double-blind, placebo-controlled phase 3 study. Lancet Neurol 2020;19:135-44

34. No authors listed. Sublingual apomorphine (Kynmobi) for Parkinson's disease. Med Lett Drugs Ther. 2020;62:165-6.

35. Caughman CY, Factor S. A critical review of apomorphine hydrochloride sublingual film for the treatment of Parkinson's disease 'OFF' episodes. Expert Rev Neurother. 2021;21:169-77.

36. Sawada $\mathrm{H}$, Oeda T, Kuno S, et al. Amantadine for dyskinesias in Parkinson's disease: a randomized controlled trial. PLoS One. 2010;5:e15298

37. Pahwa R, Tanner C, Hauser RA, et al. ADS-5102 (amantadine) extended-release capsules for levodopa-induced dyskinesia in Parkinson disease (EASE LID Study): a randomized clinical trial. JAMA Neurol. 2017:74:941-9.

38. Oertel W, Eggert K, Pahwa R, et al. Randomized, placebocontrolled trial of ADS-5102 (amantadine) extended-releas capsules for levodopa-induced dyskinesia in Parkinson's disease (EASE LID 3). Mov Disord. 2017;32:1701-9.

39. No authors listed. Osmolex ER - another extended-release amantadine for Parkinson's disease. Med Lett Drugs Ther. 2018;60:148-50

40. Perez-Lloret S, Quarracino C, Otero-Losada M, Rascol O. Droxidopa for the treatment of neurogenic orthostatic hypotension in neurodegenerative diseases. Expert Opin Pharmacother. 2019;20:635-45.

41. Palma JA, Kaufmann H. Orthostatic hypotension in Parkinson disease. Clin Geriatr Med. 2020:36:53-67.
42. Kaufmann H, Freeman R, Biaggioni I. Droxidopa for neurogenic orthostatic hypotension. A randomized, placebo-controlled, phase 3 trial. Neurology. 2014;83:328-35.

43. Biaggioni I, Freeman R, Mathias CJ, et al. Randomized withdrawal study of patients with symptomatic neurogenic orthostatic hypotension responsive to droxidopa. Hypertension. 2015;65:101-7.

44. Hauser RA, Hewitt LA, Isaacson S. Droxidopa in patients with neurogenic orthostatic hypotension associated with Parkinson's disease (NOH306A). J Parkinsons Dis. 2014;4:57-65.

45. Hauser RA, Isaacson S, Lisk JP, et al. Droxidopa for the short-term treatment of symptomatic neurogenic orthostatic hypotension in Parkinson's disease (nOH306B). Mov Disord. 2015;30:646-54.

46. Kaufmann $H$, Malamut R, Norcliffe-Kaufmann $L$, et al. The Orthostatic Hypotension Questionnaire $(\mathrm{OHQ})$ : a validation of a novel symptom assessment scale. Clin Auton Res. 2012;22:79-90.

47. Palma JA, Kaufmann H. Clinical trials for neurogenic orthostatic hypotension: a comprehensive review of endpoints, pitfalls, and challenges. Semin Neurol. 2020:40:523-39.

48. Schneider RB, lourinets J, Richard IH. Parkinson's disease psychosis: presentation, diagnosis and management. Neurodegener Dis Manag. 2017;7:365-76.

49. Martinez-Ramirez D, Okun MS, Jaffee MS. Parkinson's disease psychosis: therapy tips and the importance of communication between neurologists and psychiatrists. Neurodegener Dis Manag. 2016;6:319-30

50. Cummings J, Isaacson S, Mills R, et al. Pimavanserin for patients with Parkinson's disease psychosis: a randomised, placebocontrolled phase 3 trial. Lancet. 2014:383:533-40.

51. Schubmehl S, Sussman J. Perspective on pimavanserin and SAPS-PD: novel scale development as a means to FDA approval. Am J Geriatr Psychiatry. 2018;26:1007-11.

52. Sellers J, Darby RR, Faroogue A, Claassen DO. Pimavanserin for psychosis in Parkinson's disease - related disorders: a retrospective chart review. Drugs Aging 2019:36:647-53.

53. US Food and Drug Administration. FDA Analysis Finds No New or Unexpected Safety Risks Associated with Nuplazid (Pimavanserin), a Medication to Treat the Hallucinations and Delusions of Parkinson's Disease Psychosis. Available at: https://www.fda.gov/drugs/drug-safety-and-availability/fdaanalysis-finds-no-new-or-unexpected-safety-risks-associatednuplazid-pimavanserin-medication (accessed 19 November 2021).

54. Paff M, Loh A, Sarica C, et al. Update on current technologies for deep brain stimulation in Parkinson's disease. J Mov Disord. 2020;13:185-98

55. Schupbach WMM, Chabardes S, Matthies C, et al. Directional leads for deep brain stimulation: opportunities and challenges. Mov Disord. 2017:32:1371-5.

56. Steigerwald F, Muller L, Johannes S, et al. Directional deep brain stimulation of the subthalamic nucleus: a pilot study using a novel neurostimulation device. Mov Disord. 2016;31:1240-3

57. Pollo C, Kaelin-Lang A, Oertel MF, et al. Directional deep brain stimulation: an intraoperative double blind pilot study. Brain. 2014;137(Pt 7):2015-26.

58. Bond AE, Sha BB, Huss DS, et al. Safety and efficacy of focused ultrasound thalamotomy for patients with medicationrefractory, tremor-dominant Parkinson disease: a randomized clinical trial. JAMA Neurol. 2017;74:1412-18.

59. Eisenberg HM, Krishna V, Elias J, et al. MR-guided focused ultrasound pallidotomy for Parkinson's disease: safety and feasibility. J Neurosurg. 2020. doi. org/10.3171/2020.6.JNS192773. 